\title{
Airfoil Selection and Wingsail Design for an Autonomous Sailboat
}

\author{
Manuel F. Silva ${ }^{1,2}$, Benedita Malheiro ${ }^{1,2}$, Pedro Guedes $^{1}$, and Paulo Ferreira ${ }^{1}$ \\ ${ }^{1}$ ISEP/PPorto, School of Engineering, Polytechnic of Porto, Porto, Portugal \\ ${ }^{2}$ INESC TEC, Porto, Portugal \\ \{mss, mbm, pbg, pdf\}@isep.ipp.pt
}

\begin{abstract}
Ocean exploration and monitoring with autonomous platforms can provide researchers and decision makers with valuable data, trends and insights into the largest ecosystem on Earth. Regardless of the recognition of the importance of such platforms in this scenario, their design and development remains an open challenge. In particular, energy efficiency, control and robustness are major concerns with implications in terms of autonomy and sustainability. Wingsails allow autonomous boats to navigate with increased autonomy, due to lower power consumption, and greater robustness, due to simpler control. Within the scope of a project that addresses the design, development and deployment of a rigid wing autonomous sailboat to perform long term missions in the ocean, this paper summarises the general principles for airfoil selection and wingsail design in robotic sailing, and provides some insights on how these aspects influence the autonomous sailboat being developed by the authors.
\end{abstract}

Keywords: Rigid Wingsail, Autonomous Sailboat, Wingsail Design

\section{Introduction}

Significant research is being conducted in autonomous systems (whether they are land, marine or aerial), since these platforms are useful in a variety of tasks, due to their ability to remove humans from dangerous environments, relieve them of tedious tasks, or simply go to locations otherwise inaccessible or inhospitable [16]. Diverse applications have been envisaged for these vehicles, from exploration of remote places to warfare [26].

Concerning marine robots, most of the research has been directed to electrically or fuel powered surface and underwater vessels. This type of vehicles presents severe limitations in range and endurance, depending on battery capacity or onboard fuel for propulsion, making them unsuitable for long term operation in inaccessible areas, that would otherwise be well suited for unmanned operation. These limitations make wind propelled vessels, used by mankind since ancient times [9], into an attractive possibility. Not only do they harvest the energy for propulsion from the environment, instead of having to transport it, but also require reduced electrical power since it is just for the on board control 
systems. In such case, photovoltaic cells and/or wind generators are capable of producing enough power to run the complete electrical system, making these vehicles sustainable and autonomous in terms of energy and, therefore, capable of operating continuously, for extended periods of time, in the middle of the ocean [25]. Nonetheless, sail powered vessels also present a limitation, when compared with other forms of propulsion: if there is no wind, there is no propulsion.

Research on autonomous, or robotic, sailboats has been ongoing for about 20 to 25 years [19]. The last years have witnessed an increasing interest in the development of autonomous water surface vehicles (ASV). By robotic sailing it is meant that the whole process of sailing boat navigation is performed by an autonomously acting system of technical devices 27. The key characteristics of a robotic autonomous sailing boat are the following [27]: (i) wind is the only source of propulsion; (ii) it is not remote controlled - the entire control system is on board and, therefore, has to perform the planning and manoeuvres of sailing automatically and without human assistance; and (iii) it is completely energy self-sufficient - this is not a must in the sense of definition of a robotic sailing boat, but it opens a wider range of applications.

The use of autonomous sailboats has been proposed for different purposes, including long term oceanographic research, such as monitoring marine mammals (the absence of self-generated noise during navigation is an unique advantage of robotic sailboats for underwater acoustics applications) and automated data acquisition, surveillance of harbours, borders and other areas of interest, and as intelligent sensor buoys [25]. There are also several autonomous sailing boat prototypes developed with educational and research objectives, including the participation in robotic sailing competitions for bench marking [12/8]17].

In order to be truly autonomous, sailboats need to incorporate several distinct technologies: (i) propulsion; (ii) sensing; (iii) actuation; (iv) communication; and $(v)$ control. So far, different technologies and techniques have been proposed for each of these subsystems [25. This paper addresses specifically the propulsion system, based on a wingsail, for a new autonomous sailing vehicle.

After this introduction, Section 2 details the main characteristics of wingsails and its operation. Next, section 3 briefly introduces the hull that has been chosen for the sailboat and Section 4 describes how the airfoil for the wingsail was selected. Based on this information, Section 5 presents the design and construction of the wingsail. The paper ends with concluding remarks on Section 6

\section{Wingsail Characteristics and Operation}

This section introduces wingsails, the basic physical aspects of their operation principle, the possible options that can be considered to control its angle of attack in relation to the wind, and the operation of a tail controlled wingsail.

\subsection{Definition}

Apart from the recent sailing history, sailing boats used conventional fabric sails. This type of sails is advantageous when controlled by a human sailor [27]. By 
contrast, a wingsail is a rigid structure presenting an airfoil cross-section (like an airplane wing), which improves the lift-to-drag ratio, and being also more robust and reliable (a very important aspect for autonomous vehicles), when compared with conventional sails [5. Wingsails should not be confused with solid square sails or rigid sails. Although this concept may seem a novelty, the first rigid lift-generating devices for use as auxiliary ship propulsion were proposed and developed by Anton Flettner in 1922 [2], and, since then, several boats have been equipped with wingsails [5|2|25].

Although wingsails present some disadvantages in relation to traditional sails [25], after extensive testing with different wingsails, Neal et al. maintain that the potential gains in reliability and efficiency outweigh the problems [15].

\subsection{Operating Principle}

The primary function of the wingsail is to propel the boat through the generated lift force. The propulsion will be generated by the resulting force when the airflow interacts with the wing. The force depends on the cross-sectional shape of the wing, i.e., the airfoil.

The lift on an airfoil is primarily the result of its angle of attack $(\alpha)$, defined as the angle between the mean chord of the wing (a line drawn between the leading edge and the trailing edge of the wing) and the direction of the relative wind $\left(W_{a}\right)$. When oriented at a suitable angle, the airfoil deflects the oncoming air, resulting in a force on the airfoil in the direction opposite to the deflection. This force is known as aerodynamic force and can be resolved into two components: the component perpendicular to the direction of the apparent wind is called lift $(L)$; the component parallel to the direction of the apparent wind is called drag $(D)$. Most airfoil shapes require a positive angle of attack to generate lift, but cambered (asymmetrical) airfoils can generate lift at zero angle of attack [1]. The lift and the drag produced by an airfoil are given by Equation 1 and Equation 2. respectively, where $\rho$ represents the air density, $V$ the velocity of the wingsail, $S$ the surface of the wingsail, and $C_{L}$ and $C_{D}$ the lift and drag coefficient of the airfoil.

$$
\begin{aligned}
& L=1 / 2 \rho V^{2} S C_{L} \\
& D=1 / 2 \rho V^{2} S C_{D}
\end{aligned}
$$

Figure 1 depicts the main forces that are involved in a wingsail ASV when sailing upwind and downwind. $F$ is the aerodynamic force force that decomposes in $H$, a heeling component, and $T$, the thrust that effectively propels the vessel [6].

Due to the need to operate with the wind blowing from either side of the sailboat, wingsails tend to have a symmetrical airfoil section, although asymmetrical airfoils typically present a maximum higher lift coefficient $\left(C_{L}\right)$. To improve the characteristics of symmetrical airfoils, some wingsail arrangements include 

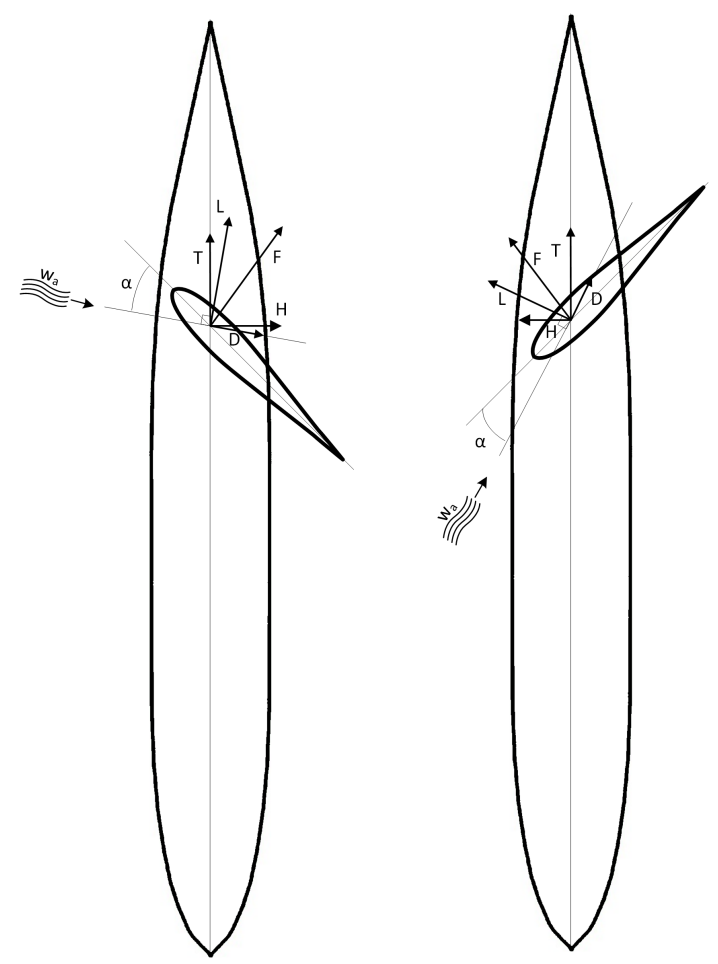

Fig. 1. Wingsail configurations for sailing upwind (left) and downwind (right) [4].

a flap or tail section to create additional lift and to help the main wing to reach its maximum lift capacity $[52]$.

\subsection{Control Architectures}

Sail control strategies in autonomous sailboats are mainly focused on controlling the wingsail angle of attack (its angle relative to the wind).

One possibility is to have the wingsail attached directly to the mast (somewhat similar to a windsurfer sail). The sail is manipulated through the rotation of the entire mast, typically using an electric motor. This design eliminates common points of failure found in traditional sails, presenting advantages for use in an autonomous sailing vessel.

There is another possibility that presents improved reliability, easier control and reduced power consumption. If the wingsail rotates freely around the mast, it will work like a wind vane, meaning it will turn to the wind without inducing large heeling moments. If the wingsail is further equipped with a smaller control surface (canard or tail wing), this arrangement (known as a tandem wing) allows the wingsail to automatically attain the optimum angle to the wind. In this situation, the wingsails are said to be self-trimming. This configuration also allows 
to adjust the angle of attack of the wingsail in relation to the wind, according to the wind speed and, thereby, vary the resulting force. This avoids other aspects of traditional sail control, such as mast raking, reefing, control of luffing and sail shape adjustment, and allows a reduction in power consumption 20].

The self-trimming capability is achieved by aligning the centre of mass of the wing arrangement on the axis of rotation (the mast). If this point is also aligned with the aerodynamic centre of the main wing, small forces from the control surface are sufficient to achieve a proper angle of attack. The aerodynamic centre on a symmetric low speed airfoil is located approximately one quarter of the chord length from the leading edge of the airfoil and characterises the position where the magnitude of the aerodynamic moment remains nearly constant for all angles of attack.

The most common tandem wing arrangement is a controllable tail behind a fully rotational wing. This transforms the fully rotational into a self-trimmed wingsail and considerably simplifies the control system design. However, this tailed wing arrangement is tail heavy, which must be compensated for with ballast positioned forward of the main wing, placing the centre of mass of the wing arrangement in the desired position. This ballast in the wing causes, on its turn, the rise of the boat's centre of gravity, making the boat more prone to capsizing, and increases the rotational radius of the wing, potentially causing damage to itself or other boats in close vicinity; however, this is not an issue out at sea where these boats usually spend most of their time apart.

A canard arrangement is similar to a tailed wing with the exception that the "tail" is in front of the main wing rather than behind. This wing arrangement has a more balanced weight placement compared to the tailed wing, meaning that a canard wing requires less ballast to position the wing's mass centre at the desired position. Also the turning radius is smaller for a canard arrangement. However, in varying wind conditions, the canard arrangement, when freely rotational, is more unstable than the tailed wing [5[2].

\subsection{Working Principle of a Tail Controlled Wingsail}

The working principle of a tail controlled wingsail is relatively simple. Typically, a wind vane is used to measure the wind direction relative to the angle of the wingsail, being this essentially a measure of the wingsail's angle of attack. While the tail has no angle of attack to the wind, the wingsail acts as a weather-vane, pointing into the relative wind, as can be seen in Figure 2 (top).

To make the sailboat move, the tail needs to be rotated to give it an angle of attack (Figure 2 (middle)), creating Lift $\left(L_{T}\right)$. This, in turn, will create a torque around the mast of the winsail $\left(T_{W}\right)$, causing the entire wingsail/tail system to rotate in the opposite direction of the tail until a torque balance is achieved between the wingsail and the tail, as depicted in Figure 2 (bottom). This moment balance will cause the wingsail to be at a positive angle of attack in relation to the apparent wind, generating lift. The component of lift pointing along the hull is the thrust, which will accelerate the sailboat until the drag on the hull equals the thrust from the wing. Once this equilibrium is achieved, the 


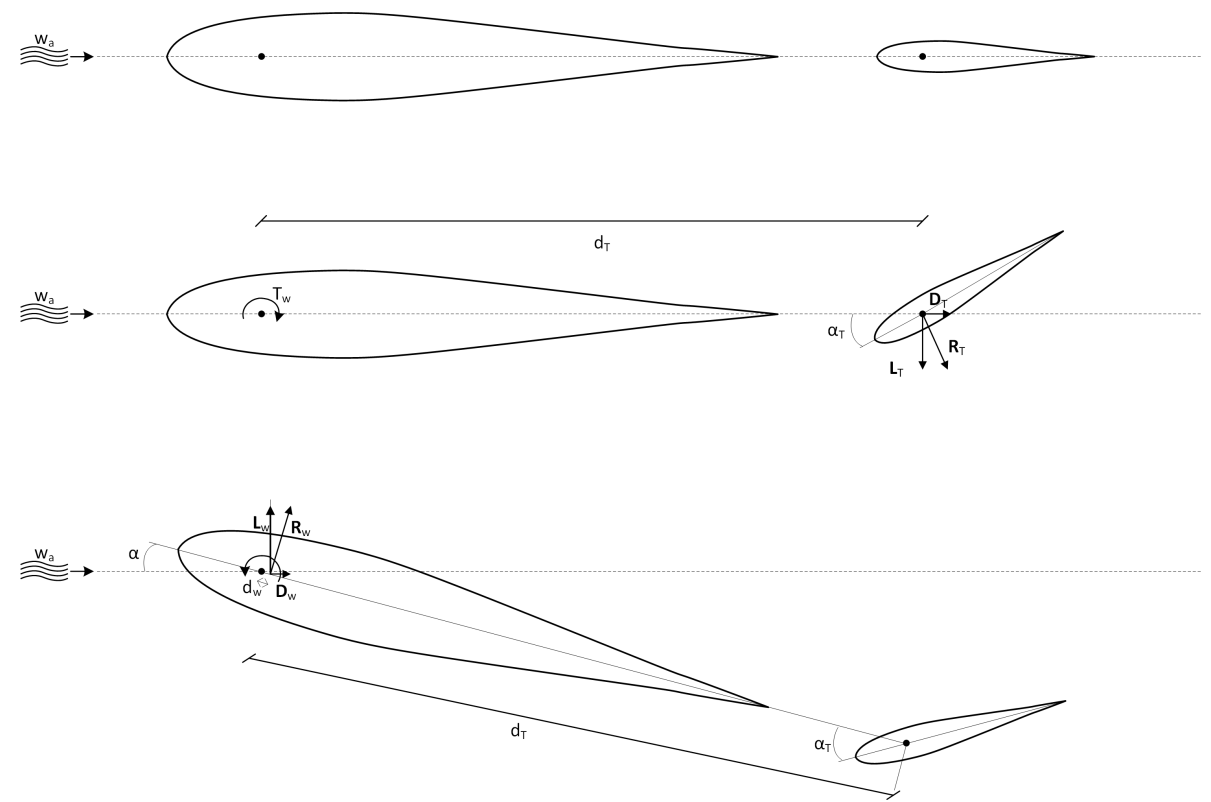

Fig. 2. Forces and torques involved in the control of the wingsail - tail set: tail not actuated (top), actuation of the tail (centre), and wingsail and tail in equilibrium (bottom).

boat will continue to move at a constant velocity, while the wing and tail are in moment equilibrium [5].

When the wind changes direction, the wingsail and tail will rotate to a new position, that is identical relative to the wind. Since the lift can only be generated perpendicular to the relative wind, the lift vector will also rotate to the same position relative to the wind. Thus, the entire wingsail, tail, and lift vector rotate together with the wind as a rigid unit [5].

The moment balance between the wing and the tail keeps the wingsail at a constant angle of attack relative to the wind. As long as the wind does not cross the centre-line of the boat, then the wing continues to provide thrust in the correct direction for forward motion through passive stability of the wingsail system. Should the wind cross through the centre-line of the boat, then the position of the flap and tails must be reversed, which corresponds to tacking or jibing, depending on whether the wind crosses the centre-line facing aft or forward, respectively [5].

\section{Sailboat Hull Selection}

Since the characteristics of the hull influence the design choices of the wingsail, this section briefly presents the options considered and the selected vessel hull. 
Several possible hull designs exist for autonomous sailing boats. Miller et al. present a series of performance trade-off studies concerning hull design features and the corresponding performance effects [13. An ideal hull would be cheap to manufacture, able to self-right in the event of capsize [7], small enough to allow for easy transportation and to prevent damage to another vessel in the event of a collision, but large enough to be able to sail effectively in heavy seas. Additionally any such hull needs to be fully enclosed to prevent water entering the hull, eliminating the need for costly pumps to remove excess water [18]. Other authors, considering the fact that this solution is very expensive to produce and not always very reliable, chose to make a completely unsinkable sailboat building it from blocks of closed cell foam [10].

Additionally, Miller et al. briefly compare monohulls with multihulls and state that catamarans and trimarans have demonstrated significantly higher performance than monohulls in many applications that do not include large changes in displacement. They argue that while the multihulls showed promise, two issues raised concerns: $(i)$ the first was weight, and (ii) the second relates to capsizing, since as multihulls are typically not self-righting, a multihull sailing boat presents a higher risk of not being able to recover from a capsize [14].

Regarding monohulls, there are three main possibilities for the hull Length Overall (LOA): $(i)$ the first one is to use a hull intended for radio controlled model boats (under $2 \mathrm{~m}$ ) [21]; (ii) a second possibility is that of a small dinghy hull ( $3 \mathrm{~m}$ to $5 \mathrm{~m}$ ); and, (iii) the final option is to modify a yacht sized hull (larger than $5 \mathrm{~m})$ [18.

The advantages of shorter vessels - option $(i)$ - are that they are cheap, lightweight, easy to handle and easy to build. Test runs can easily be arranged without the need for any special infrastructure (slip ramp, crane) or a chasing boat. On the other hand, a boat of this size is extremely sensitive even to small waves and wind gusts. This makes it difficult to reproduce experimental results and to evaluate the implications of minor changes in the control system. Furthermore, with its restricted space for additional equipment and a relatively short operating time, it is not a serious platform for maritime applications. Boats with hulls according to option (iii), mainly present the opposite characteristics. For this reason, in the last years option $(i i)$ seems to be gaining momentum with several autonomous sailboats developed based on such hulls. This is also observed when the commercial examples of rigid wingsail boats are analysed [25].

Given the above considerations, it was decided to opt for a "medium" sized monohull. After a market search, it was chosen the DCmini hull [3], depicted in Figure 3, which can be seen as an inspiration from the $2.4 \mathrm{mR}$ class. This boat, on its "traditional sail" version, presents a total sail area of $5.7 \mathrm{~m}^{2}$ distributed by the main sail with $3.4 \mathrm{~m}^{2}$ and the genoa with $2.3 \mathrm{~m}^{2}$.

\section{Airfoil Selection}

After having chosen the wingsail characteristics, it is needed to select an adequate airfoil profile to design it. 




Fig. 3. DCmini hull [3].

The Reynolds Number (Re), given by Equation 3 , represents the ratio of kinematic or inertial forces to the viscous forces in a fluid, being $\rho$ the density of the medium, $V$ the velocity of the flow, $L$ the characteristic length, and $\mu$ the viscosity of the medium.

$$
R e=\rho V L / \mu
$$

According to Elkaim, the proper Reynolds number range for sail operation is 200000 to 1.2 million [5]. Therefore, to choose the airfoil for the wingsail, the main characteristics of several symmetrical airfoils for values of $200000<R e<$ 500000 have been analysed. The objective was to find an airfoil that presented higher values for $C_{L} / C_{d}$ and $C_{L \max }$ and a relatively high maximum thickness, to improve the rigidity of the wingsail structure.

Given these selection criteria, the airfoils subjected to further consideration were the J5012, NACA 0009, NACA 64A010 and SD 8020, described in 22 23 24], CG Ultimate, DH4009, E472, Trainer 60 and Ultra-Sport 1000 [11, and several other symmetrical airfoils whose characteristics are presented in [28. The final decision fell on the Eppler E169 low Reynolds number airfoil [29], whose main aerodynamic characteristics at $R e=200000$ are depicted in the charts presented in Figure 4. From the analysis of these charts it can be concluded that this airfoil presents values of $C_{L} / C_{d} \approx 50$ for attack angles in the interval $5^{\circ}<\alpha<10^{\circ}$, while having a relatively low value of $C_{d}<0.02$ in this interval of $\alpha$. For $\alpha \approx 10^{\circ}$ the value of $C_{L} \approx 1.1$. Therefore, the wingsail should be operated with an angle of attack $\alpha \approx 10^{\circ}$ to obtain the optimal conjugation of aerodynamic parameters. This value of the angle of attack $\left(\alpha \approx 10^{\circ}\right)$ will be used in the sequel for computational purposes only and, during sailing, it will have to be adjusted to the particular course being sailed. 




(a) $C_{L} / C_{d}$ vs. $\alpha$.

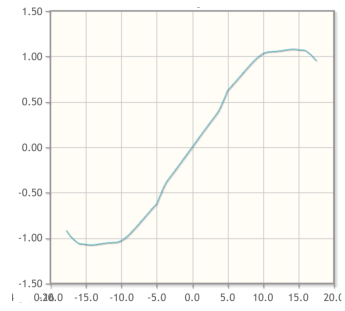

(b) $C_{L}$ vs. $\alpha$.

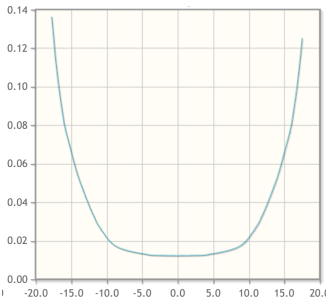

(c) $C_{d}$ vs. $\alpha$.

Fig. 4. Main aerodynamic characteristics of the Eppler E169 airfoil [29].

\section{$5 \quad$ Wingsail Design and Construction}

According to Elkaim, a sloop rig sail can achieve a maximum $C_{L} \approx 0.8$ if the jib and sail are perfectly trimmed. Realistically, with an operating maximum $C_{L} \approx 0.6$, the $C_{L} / C_{d}$ of the conventional sail is in the 3 to 5 range [5]. For the profile chosen, the ratio between the $C_{L}$ at an angle of attack $\alpha \approx 10^{\circ}$ and the $C_{L}$ for the traditional sail was computed using Equation 4

$$
C_{\text {Lratio }}=C_{\text {LE169 }} / C_{\text {Lclothsail }} \approx 1.83
$$

This value indicates that the wingsail area should be 1.83 times lower than that of the cloth sail, which would correspond to $A_{\text {wingsail }} \approx 3.1 \mathrm{~m}^{2}$. However, since the $C_{L} / C_{d}$ of the chosen profile around $\alpha \approx 10^{\circ}$ is much better that the one for the traditional sail cloth, it was decided that wingsail should have a maximum area of $A_{\text {wingsail }}=1.5 \mathrm{~m}^{2}$.

For this wingsail area, two geometrical shapes have been considered for the wing: $(i)$ a rectangular shape wingsail with an height of $1500 \mathrm{~mm}$ and a width of $1000 \mathrm{~mm}$, and (ii) a trapezoidal wingsail with an height of $1500 \mathrm{~mm}$ and a width varying between $800 \mathrm{~mm}$ to $1200 \mathrm{~mm}$. However, the current state of development of the project does not yet allows to present the polar diagrams for these two shapes of wingsails and its comparison with traditional sails

Although the trapezoidal wingsail has the advantage of having a lower centre of aerodynamic pressure for the same value of generated lift (meaning that the force that heels the boat is applied lower, lowering also the heeling torque), the decision was to build a rectangular wingsail due to its construction simplicity compared to the trapezoidal counterpart. The designed wingsail, with a wing span of $1530 \mathrm{~mm}$ and a wing chord of $1000 \mathrm{~mm}$ (Figure 5a), has a rectangular structure (Figure 5b).

The wingsail plywood ribs were cut using a computer numerical control machine. The ribs of the main sail were inserted in the mast, separated using plywood spacers and glue (Figure 5c and covered using transparent ultraviolet resistant polyvinyl chloride (Figure 5d). The assembly of the tail around a light plastic axis followed the same process. Then, the wingsail was mounted and secured on top of the frame, using two pins. The initial tests showed that tail 
rotates freely around its plastic axis and the main mast rotates around its base on the frame, moving the whole wingsail.

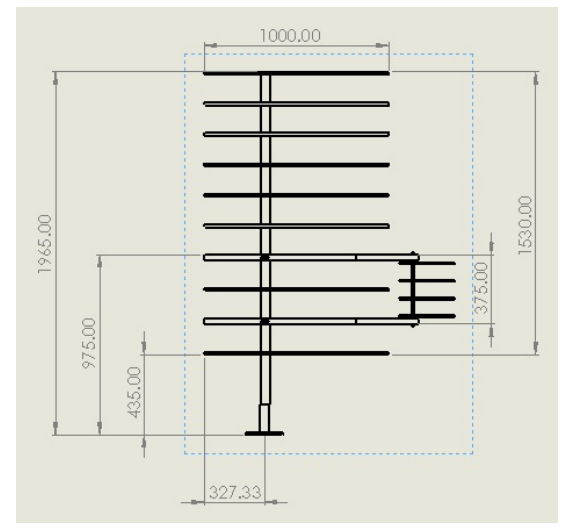

(a) Dimensions.

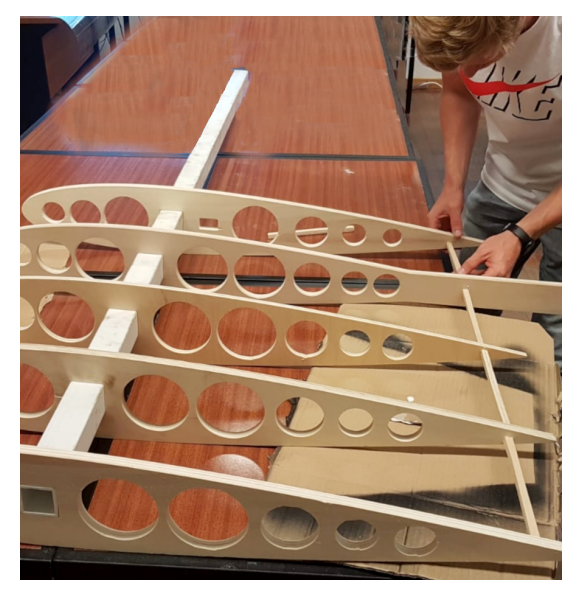

(c) Rib assembly.

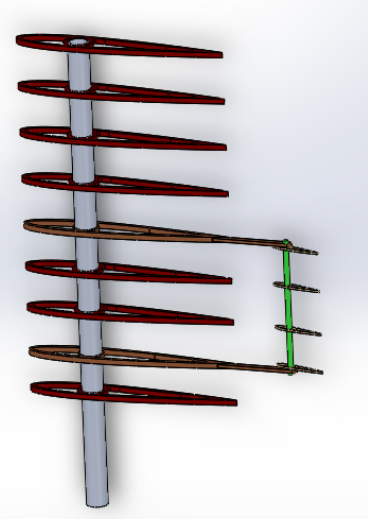

(b) Structure.

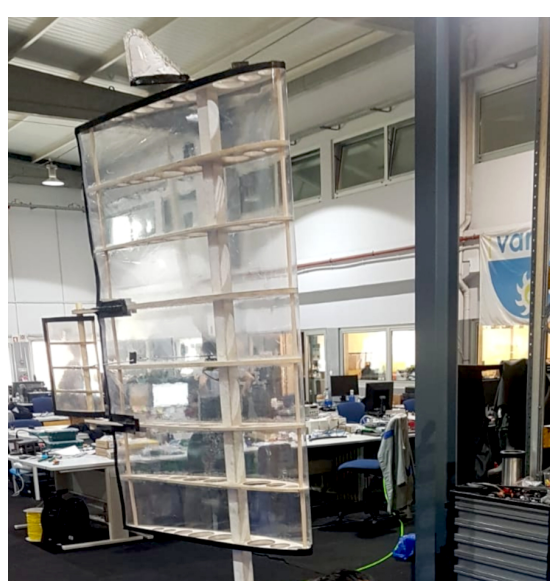

(d) Completed wingsail.

Fig. 5. Design and assembly of a rectangular tail controlled wingsail.

\section{Conclusions}

Autonomous sailing robots, and in particular the ones propelled by wingsails, present several advantages in comparison to the more common electrical or fuel propelled ASV. For this reason, the authors are developing an autonomous wingsail boat, intended for long term ocean navigation. In the scope of this project, 
this paper presented the background research performed on wingails operating principle and control followed by the decisions that led to the hull choice and the wingsail design option, including the wingsail airfoil and its dimensions. The wingsail will be submitted to performance tests in the next months.

Concerning future developments, the authors plan to address in detail the possible advantages that can arise by using a winsail with a trapezoidal shape and build a wingsail with this shape for performance comparison purposes.

\section{Funding}

This work was partially financed by National Funds through the Portuguese funding agency, Fundação para a Ciência e a Tecnologia (FCT), within project $\mathrm{UID} / \mathrm{EEA} / 50014 / 2019$.

\section{References}

1. Anderson, D.F., Eberhardt, S.: Understanding Flight. McGraw-Hill (2010)

2. Atkins, D.W.: The CFD assisted design and experimental testing of a wingsail with high lift devices. Ph.D. thesis, University of Salford (1996)

3. DelMar Conde: DCmini. https://www.delmarconde.pt/?page id=19 (2019), last Accessed: 2019-01-31

4. Domínguez-Brito, A.C., Valle-Fernández, B., Cabrera-Gámez, J., de Miguel, A.R., García, J.C.: A-tirma g2: An oceanic autonomous sailboat. In: Robotic Sailing 2015 - Proceedings of the 8th International Robotic Sailing Conference. pp. 3-13 (September 2015). DOI: 10.1007/978-3-319-23335-2_1

5. Elkaim, G.H.: System Identification for Precision Control of a WingSailed GPSGuided Catamaran. Ph.D. thesis, Stanford University (2001)

6. Elkaim, G.H., Boyce, C.L.: Experimental aerodynamic performance of a selftrimming wing-sail for autonomous surface vehicles. IFAC Proceedings Volumes 40(17), 271-276 (2007). DOI: 10.3182/20070919-3-HR-3904.00048, 7th IFAC Conference on Control Applications in Marine Systems

7. Holzgrafe, J.: Transverse stability problems of small autonomous sailing vessels. In: Robotic Sailing 2013 - Proceedings of the 6th International Robotic Sailing Conference. pp. 111-123 (September 2013). DoI: 10.1007/978-3-319-02276-5 9

8. INNOC - Österreichische Gesellschaft für innovative Computerwissenschaften: Robotic sailing. https://www.roboticsailing.org/ (2018), last Accessed: 2018-11-16

9. Kimball, J.: Physics of Sailing. CRC Press - Taylor \& Francis Group, Boca Raton, Florida (2010)

10. Leloup, R., Pivert, F.L., Thomas, S., Bouvart, G., Douale, N., Malet, H.D., Vienney, L., Gallou, Y., Roncin, K.: Breizh spirit, a reliable boat for crossing the atlantic ocean. In: Robotic Sailing - Proceedings of the 4th International Robotic Sailing Conference. pp. 55-69 (August 2011). DOI: 10.1007/978-3-642-22836-0_4

11. Lyon, C.A., Broeren, A.P., Giguère, P., Gopalarathnam, A., Selig, M.S.: Sümmary of Low-Speed Airfoil Data - Volume 3. SoarTech Publications, Virginia Beach, Virginia, USA (1997), https://m-selig.ae.illinois.edu/uiuc_lsat/ Low-Speed-Airfoil-Data-V3.pdf

12. Microtransat: The microtransat challenge. https://www.microtransat.org/index. php last Accessed: 2018-11-09 
13. Miller, P., Beeler, A., Cayaban, B., Dalton, M., Fach, C., Link, C., MacArthur, J., Urmenita, J., Medina, R.Y.: An easy-to-build, low-cost, high-performance sailbot. In: Robotic Sailing 2014 - Proceedings of the 7th International Robotic Sailing Conference. pp. 3-16 (September 2014). Dor: 10.1007/978-3-319-10076-0_1

14. Miller, P.H., Hamlet, M., Rossman, J.: Continuous improvements to usna sailbots for inshore racing and offshore voyaging. In: Robotic Sailing 2012 - Proceedings of the 5th International Robotic Sailing Conference. pp. - (September 2012). DOI: 10.1007/978-3-642-33084-1 5

15. Neal, M., Sauzé, C., Thomas, B., Alves, J.C.: Technologies for autonomous sailing: Wings and wind sensors. In: Proceedings of the 2nd International Robotic Sailing Conference. pp. 23-30 (July 2009)

16. Olson, S. (ed.): Autonomy on Land and Sea and in the Air and Space: Proceedings of a Forum. The National Academies Press, Washington, DC (2018). Dor:10.17226/ 25168

17. SailBot: Sailbot | international robotic sailing regatta. https://www.sailbot.org/ (2018), last Accessed: 2018-11-16

18. Sauzé, C., Neal, M.: An autonomous sailing robot for ocean observation. In: Proceedings of the 7th Towards Autonomous Robotic Systems (TAROS) Conference. pp. 190-197 (September 2006)

19. Sauzé, C., Neal, M.: Design considerations for sailing robots performing long term autonomous oceanography. In: Proceedings of the International Robotic Sailing Conference. pp. 21-29 (May 2008)

20. Sauzé, C., Neal, M.: Moop: A miniature sailing robot platform. In: Robotic Sailing Proceedings of the 4th International Robotic Sailing Conference. pp. 39-53 (August 2011). DOI: 10.1007/978-3-642-22836-0_3

21. Schlaefer, A., Beckmann, D., Heinig, M., Bruder, R.: A new class for robotic sailing: The robotic racing micro magic. In: Robotic Sailing - Proceedings of the 4th International Robotic Sailing Conference. pp. 71-84 (August 2011). DOI: 10.1007/978-3-642-22836-0 5

22. Selig, M.S., Donovan, J.F., Fraser, D.B.: Airfoils at low speeds. H. A. Stokely, Virginia Beach, Virginia, USA (1989), https://m-selig.ae.illinois.edu/uiuc_lsat/ Airfoils-at-Low-Speeds.pdf

23. Selig, M.S., Guglielmo, J.J., Broeren, A.P., Giguère, P.: Summary of Low-Speed Airfoil Data - Volume 1. SoarTech Publications, Virginia Beach, Virginia, USA (1995), https://m-selig.ae.illinois.edu/uiuc_lsat/Low-Speed-Airfoil-Data-V1.pdf

24. Selig, M.S., Lyon, C.A., Giguère, P., Ninham, C.P., Guglielmo, J.J.: Summary of Low-Speed Airfoil Data - Volume 2. SoarTech Publications, Virginia Beach, Virginia, USA (1996), https://m-selig.ae.illinois.edu/uiuc lsat/ Low-Speed-Airfoil-Data-V2.pdf

25. Silva, M.F., Friebe, A., Malheiro, B., Guedes, P., Ferreira, P., Waller, M.: Rigid wing sailboats: A state of the art survey. Ocean Engineering 187, 106-150 (2019), http://www.sciencedirect.com/science/article/pii/S0029801819303294

26. Springer, P.J.: Outsourcing War to Machines - The Military Robotics Revolution. Praeger Security International, Santa Barbara, California (2018)

27. Stelzer, R.: Autonomous Sailboat Navigation - Novel Algorithms and Experimental Demonstration. Ph.D. thesis, De Montfort University (2012)

28. Tools, A.: Airfoil tools. http://airfoiltools.com/ (2018), last Accessed: 2019-02-04

29. Tools, A.: Airfoil tools. http://airfoiltools.com/airfoil/details?airfoil=e169-il\# polars (2018), last Accessed: 2019-02-04 